\title{
Silvia Kutscher
}

\section{Emotionsverben im Estnischen: Konstruktionsvarianz und Kausalstruktur}

The article deals with the constructional variation of emotion predicates in Estonian. It gives an overview of constructional types, including information on their quantitative distribution. I show that one characteristic of Estonian is the formation of pairs of converses, i.e. paired emotion verbs, which have the same emotional semantics but different argument realisation patterns. These converses are based on derivational morphology, such as the causative morpheme - $t a$ 'CAUS'. Causative derivation has been adduced in the theoretical literature as support for the assumption that the cross-linguistically widespread constructional variation in emotion predicates has its origin in a difference in the causal structure in verbal semantics. The article shows that the data for Estonian contradict this assumption.

\section{Einleitung}

Aus der Forschungsliteratur ist hinlänglich bekannt, dass sich übereinzelsprachlich und einzelsprachlich die semantische Klasse der Emotionsverben hinsichtlich ihrer konstruktionellen Bandbreite von der Klasse der kanonischen Handlungsverben - also Verben, die Handlungen im physischen Raum denotieren, die von einem Agens ausgeübt werden und auf ein Patiens gerichtet sind (z. B. dt. etwas zerbrechen, jmdn. töten, etwas erbauen) unterscheidet. Während kanonische Handlungsverben sprachintern einem einheitlichen Konstruktionstyp (in Akkusativsprachen ist dies Agens in Subjektposition bzw. im Nominativ und Patiens in Objektposition bzw. im Akkusativ) angehören, s. (1a), weisen Emotionsverben in vielen Sprachen eine Konstruktionsvariation auf. So kennt z. B. das Deutsche u. a. die in (1b) bis (1d) angeführten Argumentrealisierungstypen für Emotionsverben.
a. Er schlägt dich.
(Ag/NOM\&Pat/AKK)
b. Er mag dich.
(Exp/NOM \&Stim/AKK)
c. Du beeindruckst ihn.
(Exp/AKK \&Stim/NOM)
d. Du gefällst ihm.
(Exp/DAT\&Stim/NOM ) 
Geht man davon aus, dass Emotionsverben grundsätzlich über zwei semantische Rollen verfügen, nämlich die des Wahrnehmenden (Experiencer) und die des Wahrgenommenen bzw. des Auslösenden des durch das Verb bezeichneten affektiven Zustandes (Stimulus), so stellen die Emotionsverben für jedes theoretische Modell zur Argumentrealisierung insofern einen Prüfstein dar, als sie typischerweise auf der syntaktischen Ebene in Basiskonstruktionen eine Konstruktionsvielfalt aufweisen, die nicht direkt über die Verknüpfung mit den beiden semantischen Rollen von Emotionsverben motiviert werden kann, wie (1) für das Deutsche demonstriert.

Im Unterschied zum älteren rein syntaktischen Ansatz (Postal 1971; Beletti/Rizzi 1988) gehen die meisten neueren Theorien zur Argumentrealisierung bei Emotionsverben von einer semantischen (u. a. Van Valin/LaPolla 1997; Dowty 1991; Grimshaw 1990; Pesetsky 1995) oder kognitiven Basis aus (u. a. Croft 1991, 1993), auf der der Mechanismus der Argumentrealisierung operiert. Dabei ist für diese Modelle die Frage nach der Implikation einer Kausalrelation für die Motivierung der Argumentrealisierung bei Emotionsverben besonders relevant. Von prominenten Vertretern dieser Forschungsrichtung (z. B. Grimshaw 1990; Pesetsky 1995; Iwata 1995) werden Emotionsverben aufgrund von Unterschieden in ihrer Kausalstruktur in zwei Klassen aufgeteilt, nämlich in die Klasse von Verben, deren Experiencer als Subjekt realisiert wird (Subjekt-Experiencer-Verben) und in die, deren Experiencer als Objekt realisiert wird (Objekt-Experiencer-Verben). Nach dieser Auffassung denotieren die Objekt-Experiencer-Verben (z. B. engl. she pleases him) im Gegensatz zu den Subjekt-Experiencer-Verben (z. B. engl. he likes her) einen vom Stimulus kausierten, patienstypischen Zustandswechsel des Experiencers. Die Realisierung des Stimulus als Subjekt wird durch seine Rolleneigenschaft als Verursacher des mentalen Zustands beim Experiencer erklärt. Zur Unterstützung dieser auf einem Kausalitätsunterschied basierenden Motivierung der Argumentrealisierungsvarianz bei Emotionsverben werden in einigen einschlägigen Arbeiten Daten aus Sprachen mit verbaler Kausativmorphologie herangezogen. Dabei wird argumentiert, dass in Sprachen mit overter Kausativkennzeichnung die Objekt-Experiencer-Verben im Unterschied zu den Subjekt-ExperiencerVerben Kausativmorphologie aufweisen (Grimshaw 1991: 23; Pesetsky 1995: 63-69; Van Valin/LaPolla 1997: 98). Das Estnische ist ebenfalls eine Sprache mit verbaler Kausativmorphologie, wurde bisher jedoch nicht im Hinblick auf die Distribution der Kausativderivation bei Emotionsverben untersucht. Diese Forschungslücke versucht die vorliegende Arbeit zu schließen. 
Im Folgenden werden die im Estnischen belegten Konstruktionstypen der Emotionsverben im von mir zusammengestellten Korpus dargestellt. In einem weiteren Abschnitt erfolgt eine ausführliche Untersuchung der Distribution der Derivationsmorphologie über die Konstruktionstypen.

In den einschlägigen Arbeiten zur Konstruktionsvarianz bei Emotionsverben beschränkt sich die Analyse in der Regel auf einige wenige Verben. Dies hat sich für die Erforschung der Argumentrealisierung bei Emotionsverben als problematisch erwiesen, da z. B. Konstruktionstypen einer Sprache nicht vollständig erfasst werden (wie etwa in den Arbeiten von Härtl (1999, 2001) zum Deutschen, in der die Konstruktionstypen Exp/DAT und Stim/DAT fehlen), oder unzutreffende Verallgemeinerungen zum grammatischen Verhalten eines Konstruktionstyps gemacht werden (vgl. dazu die Diskussion zu deutschen Emotionsverben des Typs Exp/AKK in Klein/ Kutscher (2002: 7-11; 2005: 5-10)). Um ähnliche Fehlanalysen für das Estnische zu vermeiden, wurde für die vorliegende Untersuchung ein Korpus mit 110 Verben zusammengestellt. ${ }^{1}$

Die für die vorliegende Arbeit zusammengestellten Emotionsausdrükke stammen aus zweisprachigen (Kann et al. 1972; Kibbermann/Kirotar/ Koppel 1975, abgekürzt als (Kibbermann); Saagpakk 1992; Mägiste 1983) und einsprachigen Wörterbüchern (Raiet et al. 1988-2002, abgekürzt als (EKSS)) sowie einem Valenzwörterbuch (Pool 1999). Die in den folgenden Abschnitten angeführten Sprachbeispiele stammen sowohl aus den verwendeten Wörterbüchern als auch aus zwei von mir ausgewerteten literarischen Quellen, Tere kollane kass, einem Roman von Mati Unt (abgekürzt als MU) und Väike prints, einer Übersetzung der Erzählung Le Petit Prince von Antoine de Saint-Exupéry (abgekürzt als VP). Die Beispiele aus englischsprachigen Quellen wurden von mir übersetzt. Die Glossierungen zu allen angeführten Beispielen stammen von mir.

\section{Verbale Derivation im Estnischen}

Das Estnische verfügt über drei Teilsysteme verbaler Derivation. Das erste Teilsystem dient der Derivation verschiedener Aktionsarten (s. beispielhaft (2)) und ist für die vorliegende Arbeit von untergeordnetem Interesse. 
(2) -tse 'durativ':

nokki-ma 'picken, hacken' $\rightarrow$ noki-tse-ma 'im Garten werkeln'

$-(t) l e$ 'frequentativ':

tege-ma 'tun, machen' $\rightarrow$ tege-le-ma 'sich beschäftigen'

-ata 'momentan':

karju-ma 'schreien' $\rightarrow$ karj-ata-ma 'aufschreien'

Des weiteren können die beiden Derivationstypen der interkategoriellen Nomen-zu-Verb-Derivation und die intrakategorielle valenzverändernde Derivation von Verben unterschieden werden (vgl. z. B. Kasik 1997: 43)². Die sogenannten interkategoriellen Suffixe zur Ableitung von Verben aus nominalen Wurzeln sind jedoch formgleich mit sowohl aktionsartenderivationellen (3a) als auch valenzverändernden (s. u. (4) - (7)) Derivationssuffixen $(3 \mathrm{~b}, 3 \mathrm{c})$. Die Unterscheidung zwischen inter- und intrakategorieller Derivation ist daher nur auf semantisch-funktionaler Ebene und nicht hinsichtlich der morphologischen Form zu treffen. Aufgrund der Formgleichheit wurde daher für die vorliegende Arbeit eine einheitliche Glossierung der entsprechenden Morpheme gewählt. ${ }^{3}$

(3) a. staari-tse-ma

Star-DUR-INF

'sich wie ein Star aufführen' (Kasik 1997: 58)

b. all-u-ma

unten-REFL-INF

‘jmdm. untergeordnet sein / sich unterordnen’ (Kasik 1997: 54)

c. rikas-ta-ma

reich-KAUS-INF

'reich machen' (Kasik 1997: 55)

Von den derivierten verbalen Stämmen können über modifizierende oder valenzverändernde Derivationsaffixe neue Verben abgeleitet werden. Anders als im Finnischen (s. Pylkkänen 2000; Nelson 2003) ist die verbale Derivation allerdings nicht-rekursiv, $d$. h. jeder oben illustrierte Derivationstyp kann im Estnischen nur einmal pro Stamm durchgeführt werden. 


\title{
Silvia Kutscher
}

Daher ist eine verbale Derivationskette im Estnischen auf höchstens drei Derivationsschritte beschränkt (Kasik 1997: 54f.). Von einem monosyllabischen nominalen Stamm wird in einem ersten Schritt durch Derivation mit dem Morphem - $u$ ein einstelliges Verb deriviert, s. (3b). Von diesem kann in einem zweiten Schritt durch Derivation mit dem Morphem -ta ein zweistelliges Verb deriviert werden (4a). Von einem polysyllabischen nominalen Stamm wird zunächst durch KAUS-Derivation ein zweistelliges Verb (3c) und in einem zweiten Schritt kann durch Affigierung mit dem Morphem - $u$ 'REFL' ein einstelliges Verb deriviert werden (4b). Dabei kommt es zur lautlichen Verkürzung des Kausativaffixes durch Ausfall des Vokals /a/.

\author{
(4) a. all-u-ta-ma \\ unten-REFL-KAUS-INF \\ ‘jmdn. unterwerfen, erniedrigen' (Kasik 1997: 54) \\ b. rikas-t-u-ma \\ reich-KAUS-REFL-INF \\ 'reich werden' (Kasik 1997: 55)
}

Die Kategorie des Kausativs wird am Verbstamm durch das Affix - $t a$ bzw. eines seiner Allomorphe (-t, $-t t a,-d a,-a)$ gekennzeichnet und ist entweder von intransitiven Verben (5a) oder von einem nominalen Stamm (5b) abgeleitet (Viitso 2003: 69).

(5) a. keema 'kochen (itr.)' $\rightarrow \quad$ kee-t-ma 'kochen (tr.)'

$\begin{array}{llll}\text { Piim } & \text { kee- } b . & \text { Ta kee-da-b } & \text { piima. } \\ \text { Milch:NOM } & \text { koch -PRS:3SG } & \text { 3SG koch-KAUS-PRS:3SG } & \text { Milch:PAR } \\ \text { 'Die Milch kocht.' } & \text { 'Er/Sie kocht die Milch. } & \end{array}$

b. riie 'Kleidung, Stoff' $\quad \rightarrow \quad$ riie-ta-ma 'jmdn. ankleiden'

\begin{tabular}{|c|c|c|}
\hline Ema & riie-ta-s & last \\
\hline Mutter & kleid-KAUS-PRÄT:3SG & Kind:PAR \\
\hline
\end{tabular}

Denominale -ta-Ableitungen können in transitive (6a) oder intransitive Verben (6b) resultieren. Sie bezeichnen einen Prozess, der das von der no- 
minalen Basis bezeichnete Objekt affiziert oder effiziert (Kausativ im engeren Sinne), s. (6a), bzw. ausdrückt, dass jemand mit dem von der nominalen Basis denotierten Objekt ausgestattet wird (Instruktiv) bzw. das denotierte Objekt herstellt bzw. verändert (Faktitiv), s. (6b). ${ }^{4}$
a. kirju-ta-ma 'schreiben' $\leftarrow$ kiri 'Schriftstück, Brief's
Kirjutan
magistritööd
schreib-KAUS-PRS:1SG Magisterarbeit:PAR
'Ich schreibe an meiner Magisterarbeit.'
b. vale-ta-ma 'lügen' $\leftarrow$ vale 'Lüge'

$\begin{array}{lllll}\text { Kes } & \text { vale-ta- } b, & \text { see } & k a & \text { varastab } \\ \text { wer:NOM lüg-KAUS-PRS:3SG } & \text { DEM } & \text { auch } & \text { stehl-PRS:3SG } \\ \text { 'Wer lügt, stiehlt auch.' (Saagpakk } & \text { 1992: } & \text { 1054, Lemma valetaja) }\end{array}$

Die denominalen -ta-Ableitungen enthalten in diesem Sinne semantisch ebenfalls eine Kausativkomponente. ${ }^{6}$ Im Weiteren werde ich daher auch die denominalen - $t a$-Ableitungen verallgemeinert als Kausativ bezeichnen und Faktitiva und Instruktiva als semantische Subtypen der morphologisch definierten Gruppe der Kausativa auffassen. ${ }^{7}$

Die Valenzreduktion eines Verbs ist formal durch das Affix $-u$ bzw. eines seiner morphophonologisch bedingten Allomorphe (- $d u,-b u,-b i)$ gekennzeichnet. Das Affix steht sowohl mit solchen Sachverhaltsausdrücken, die eine Handlung denotieren, die ein Agens mit sich selbst ausführt ( $7 \mathrm{a}$ ), als auch mit Vorgangsausdrücken, die ohne kontrollierenden Agens konzeptualisiert sind ( $7 \mathrm{~b}){ }^{8}$

(7) a. viska-ma 'werfen' $\rightarrow \quad$ visk-u-ma 'sich werfen'

$\begin{array}{lll}\text { Ta } & \text { viska-s } & \text { kivi } \\ \text { 3SG } & \text { werf-PRÄT:3SG } & \text { Stein:GEN } \\ \text { 'Sie/Er warf den Stein.' } & \\ & & \\ \text { Ta } & \text { visk-u-s } & \text { voodi-sse } \\ \text { 3SG } & \text { werf-REFL-PRÄT:3SG } & \text { Bett-ILL } \\ \text { 'Sie/Er warf sich aufs Bett'. (Saagpakk 1992: 1103) }\end{array}$


b. murdma 'zerbrechen (tr.)' $\rightarrow$ murd-u-ma 'zerbrechen (itr)'

Murd-si-n šokolaaditahvli tükki-de-ks

zerbrech-PRÄT-1SG Schokoladentafel:GEN Stück-PL-TRA

'Ich zerbrach die Schokoladentafel in Stücke.' (EKSS: 502)

$\begin{array}{llll}\text { Aer } & \text { murdu-s } & \text { järsu-st } & \text { tõmbe-st } \\ \text { Ruder:NOM } & \text { zerbrech-PRÄT:3SG } & \text { tief-ELA } & \text { Zug-ELA } \\ \text { 'Das Ruder zerbrach durch den kräftigen Zug.' } & \text { (EKSS: 504) }\end{array}$

Zur Argumentrealisierung bei Emotionsverben liegen öffentlich zugänglich keine speziellen Arbeiten in der linguistischen Literatur zum Estnischen vor ${ }^{9}$, es gibt eine eher ethnopsychologisch ausgerichtete Studie zum Emotionswortschatz des Estnischen (Vainik 2001, 2002), die sich auf die Einschätzung von Gefühlswerten und die statistische Erhebung von Nennungs- und Verwendungshäufigkeiten mehrheitlich nominaler Ausdrücke für Emotionen im Estnischen konzentriert.

\section{Zu den Argumentrealisierungstypen der Emotionsverben}

Das Estnische verfügt über eine große Zahl verschiedener Konstruktionstypen für Emotionsausdrücke. Tabelle (1) stellt diese überblicksartig dar. Neben Emotionsverben finden sich im Estnischen Körperteilkonstruktionen und Konstruktionen, in denen der Empfindungszustand durch ein Nominal denotiert wird. Da diese nicht Gegenstand der vorliegenden Untersuchung sind, wurden sie nicht in die tabellarische Übersicht aufgenommen. 
Emotionsverben im Estnischen: Konstruktionsvarianz und Kausalstruktur

\begin{tabular}{|l|l|l|l|}
\hline \multicolumn{1}{|c|}{ Exp } & NOM & PAR & LOK \\
\hline kein & ägestuma 'aufbrausen' & - & - \\
\hline NOM & - & $\begin{array}{l}\text { hirmutama } \\
\text { 'ängstigen' }\end{array}$ & $\begin{array}{l}\text { meeldima } \\
\text { 'gefallen' }\end{array}$ \\
\hline PAR & $\begin{array}{l}\text { imetlema 'bewundern' } \\
\text { armastama 'lieben' }\end{array}$ & - & - \\
\hline LOK & $\begin{array}{l}\text { armuma 'sich verlieben' } \\
\text { huvituma 'sich interessieren' }\end{array}$ & - \\
\hline PP & $\begin{array}{l}\text { muretsema pärast } \\
\text { 'sich beunruhigen über' } \\
\text { rõómutsema üle 'sich freuen über' }\end{array}$ & - & - \\
\hline
\end{tabular}

Tabelle (1): Konstruktionstypen der Emotionsverben im Estnischen

Wie aus Tabelle (1) ersichtlich ist, können Emotionsverben im Estnischen sowohl einstellige als auch zweistellige Prädikate bilden. Einstellige Emotionsprädikate haben im Estnischen immer ein Experiencer-Argument und regieren den Nominativ, s. (8).

(8) Algu-l ol-i ta õige tõsine,

Anfang-ADE sei-PRÄT:3SG 3SG:NOM recht ernst:NOM

hiljem lõbustu-s

später amüsier-PRÄT:3SG

'Anfänglich war er/sie recht ernst, später amüsierte sie/er sich.' (EKSS: 226)

Ausdrücke, die neben einem Experiencer im Nominativ auch einen Stimulus-Partizipanten enthalten, verteilen sich auf verschiedene Konstruktionstypen, wie die folgenden Erörterungen zeigen werden.

\subsection{Exp/NOM}

\section{- Exp/NOM\&Stim/PAR}

Der erste Konstruktionstyp ist der in (9a) illustrierte: der Experiencer steht im Nominativ, der Stimulus-Partizipant wird durch eine NP im Partitiv repräsentiert. (9b) gibt weitere Beispiele für Verben diesen Konstruktionstyps aus dem vorliegenden Korpus. 


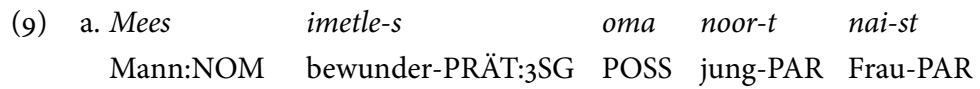

'Der Mann bewunderte seine junge Frau.' (Pool 1999: 31)

b. Exp/NOM\&Stim/PAR-Verben:

armastama 'lieben', austama 'respektieren', häbenema 'sich einer Sache schämen', jälestama 'verabscheuen', kadestama 'beneiden', kahetsema 'bedauern', kartma 'fürchten', nautima 'genießen', pelgama 'fürchten, zurückscheuen', põlgama 'verachten', (umb)usaldama '(miss)trauen', vihkama 'hassen', võorrastama 'fremdeln',

\section{- Exp/NOM\&Stim/LOK}

Bei einer im Korpus gleich großen Gruppe von Prädikaten wird der Stimulus-Partizipant durch eine Lokalkasus-NP, vgl. (12), oder durch eine PP, vgl. (10), repräsentiert. Die Emotionsverben regieren dabei unterschiedliche Postpositionen, z. B. üle 'über' (10a) oder pärast 'nach' (1ob).

(10) a. Sügise tuleku üle ei rõõmutse ta sugugi Frühling:GEN Ankunft:GEN über NEG freu:KONNEG 3 SG überhaupt 'Über die Ankunft des Frühlings freute sie/er sich überhaupt nicht.' (EKS)

b. Minu pärast ei ole vaja muretse-da 1SG:GEN nach NEG sei:KONNEG nötig sich.sorg-INF

'Es ist nicht notwendig, sich um mich zu sorgen.' (Pool 1999: 66)

Die in (11) aufgelisteten Verben sind weitere Beispiele diesen Konstruktionstyps.

(11) Exp/NOM\&Stim/PP-Verben:

igatsema järele 'sich sehnen nach', imestama üle 'sich wundern über', ägestuma pärast 'aufbrausen' nördima üle 'sich entrüsten über', pahanduma peale 'sich ärgern über', vihastuma üle 'sich erzürnen über', ...

Bei den Lokalkasus sind sowohl direktionale (12a) als auch ablativische (12b) und lokativische (12c) Kasusformen vertreten. Die Liste in (12d) gibt weitere Verben diesen Konstruktionstyps. 
(12) a. Ta mäge-de-sse juba lapse-na 3SG:NOM verlieb-PRÄT:3SG Berg-PL-ILL schon Kind-ESS 'Sie/Er verliebte sich schon als Kind in die Berge'. (Pool 1999: 14)

b. Mart huvit-u-b spordi-st Mart:NOM interessier:PRS-REFL-3SG Sport-ELA 'Mart interessiert sich für Sport.' (Pool 1999: 28)

c. Õppejõud pett-u-s üliōpilase-s Lehrkraft:NOM entäusch-REFL-PRÄT:3SG Student-INE 'Die Lehrkraft war von ihrem Studenten enttäuscht'. (Pool 1999: 79)

d. Exp/NOM\&Stim/LOK-Verben: ärrituma millest 'irritiert sein von', hämmastuma millest 'überrascht sein von', innustuma millest 'in Begeisterung geraten über', kiinduma millesse 'hängen an', rõõmustuma millest 'sich freuen über', tüdinema millest 'überdrüssig werden', üllatuma millest 'überrascht von', ...

Die innere Lokalkasusreihe scheint sich als Kennzeichnung für den Stimulus bei Emotionsverben durchzusetzen. Nur wenige Verben in meinem Korpus regieren den Allativ, s. lootma 'hoffen' in (13).

(13) Kas ma võin sinule loot-a?

QU 1SG könn-1SG 2SG-ALL hoff-INF

'Can I rely on you?' (Pool 1999: 59)

\subsection{Stim/NOM}

\section{- Stim/NOM\&Exp/PAR}

Nach der Exp/NOM\&Stim/PAR-Konstruktion ist die umgekehrte Konstruktion mit Stimulus-Partizipant im Nominativ und Experiencer-Partizipant im Partitiv (s. (14)) die zweithäufigste in meinem Korpus.
(14) Koera
hauku-mine
ehma-ta-s
last.
Hund:GEN bell-NOM'er:NOM
erschreck-KAUS-PRÄT:3SG
Kind:PAR
'The dog's barking startled the child.' (Pool 1999: 20) 
In (15) sind weitere Verben diesen Konstruktionstyps beispielhaft angeführt.

(15) Exp/PAR\&Stim/NOM-Verben:

erutama 'erregen', häirima 'stören', hämmastama 'erstaunen', hirmutama 'erschrekken', huvitama 'interessieren', innustama 'begeistern', julgustama 'ermutigen', kurvastama 'betrüben', liigutama 'bewegen', lôbustama 'erfreuen', pahandama 'verärgern', peletama 'einschüchtern', rahustama 'beruhigen', rahuldama 'zufriedenstellen', segama 'nerven', tülitama 'verärgern', üllatama 'überraschen', vihastama 'erzürnen', ärritama 'irritieren',...

\section{- Stim/NOM\&Exp/LOK}

Der Konstruktionstyp Stim/NOM\&Exp/LOK ist im Korpus ebenfalls vertreten, beschränkt sich aber im Gegensatz zur Konstruktion Exp/ NOM\&Stim/LOK auf ein Verb, meeldima 'mögen, gefallen' mit Allativ (-le), s. (16). Dieses Verb ist allerdings eines von hoher Verwendungsfrequenz im Estnischen.
(16) Liisale meeldi-vad looma-d.
Liisa-ALL gefall:PRS-3PL Tier-PL
'Liisa mag Tiere.' (Pool 1999: 64)

Zusammenfassend ergibt sich, dass das Estnische bezüglich der Argumentrealisierung bei Emotionsverben eine Vielzahl von Konstruktionstypen aufweist, welche überblicksartig noch einmal in Tabelle (2) aufgelistet werden.

Exp/NOM-Konstruktionen: Stim/NOM-Konstruktionen:

$\operatorname{Exp} / \mathrm{NOM}$

Exp/NOM\&Stim/PAR

Exp/NOM\&Stim/LOK

$\operatorname{Exp} / \mathrm{NOM}(\& \operatorname{Stim} / \mathrm{PP})$

$$
\begin{aligned}
& \text { *Stim/NOM } \\
& \text { Stim/NOM\&Exp/PAR } \\
& \text { Stim/NOM\&Exp/LOK } \\
& \text { *Stim/NOM }(\& E x p / P P)
\end{aligned}
$$

Tabelle (2): Argumentrealisierungstypen bei Emotionsverben des Estnischen [* = im Korpus nicht belegter Konstruktionstyp, () = syntaktisch fakultativ] 
$\mathrm{Zu}$ beachten ist, dass es keine Emotionsverben gibt, die nur ein StimulusArgument realisieren, während es umgekehrt eine Reihe von Emotionsverben gibt, deren einziges Argument ein Experiencer ist. Gleichzeitig ist die Kennzeichnungsbandbreite für den Experiencer kleiner als für den Stimulus, da er im wesentlichen auf die Realisierung im NOM oder PAR beschränkt ist und nur marginal (1 Beleg: meeldima 'gefallen', s. Beispiel (16)) die Realisierung als LOK-Argument erlaubt. Wie in den obigen Ausführungen angedeutet, verteilen sich die Konstruktionsmuster quantitativ nicht gleichmäßig über das von mir erstellte Korpus an Emotionsverben des Estnischen. Vielmehr ist ein deutlicher Schwerpunkt bei den in Tabelle (3) aufgelisteten drei der sechs insgesamt belegten Konstruktionstypen zu verzeichnen, auf die sich insgesamt ca. 90\% aller Emotionsverben des Korpus verteilen. Eine valide statistische Aussage müsste allerdings auf einem genau definierten, möglichst viele der estnischen Emotionsverben umfassenden Korpus basieren. Die in den Tabellen (3) und (4) dargestellten Zahlenverhältnisse sind dementsprechend als impressionistische Vorstudie zur quantitativen Verteilung der Konstruktionsvarianten im estnischen Emotionswortschatz zu lesen.

Exp/NOM\&Stim/PAR $\rightarrow$ ca. $30 \%$ der Verben im Korpus (35 von 110)
Exp/NOM\&Stim/LOK $\rightarrow$ ca. 30\% der Verben im Korpus (35 von 110)
Exp/PAR\&Stim/NOM $\rightarrow$ ca. $25 \%$ der Verben im Korpus (30 von 110)

Tabelle (3): prozentuale Verteilung der drei häufigsten Konstruktionstypen im Korpus

Bezogen auf die generalisierten Typen Exp/NOM (Subjektexperiencerverben) und Exp/nicht-NOM (Objektexperiencerverben) dominieren erstere im Korpus prozentual mit ca. 70\% gegenüber ca. 30\%, vgl. Tabelle (4). 


\begin{tabular}{|llll|}
\hline Konstruktionstyp & & \multicolumn{2}{l|}{ Anzahl der Verben } \\
\hline Exp/NOM & (\&Stim/Adjunkt $)$ & $>10 \%$ & $(10 / 110)$ \\
\hline & \&Stim/PAR & $30 \%$ & $(35 / 110)$ \\
\hline Summe & \&Stim/LOK & $30 \%$ & $(35 / 110)$ \\
\hline
\end{tabular}

\begin{tabular}{|llll|}
\hline Konstruktionstyp & & \multicolumn{2}{l|}{ Anzahl der Verben } \\
\hline Exp/nicht-NOM & Exp/PAR\&Stim/NOM & $30 \%$ & $(30 / 110)$ \\
\hline & Exp/LOK \&Stim/NOM & $>1 \%$ & $(1 / 110)$ \\
\hline Summe & & ca. 30\% & $(31 / 110)$ \\
\hline
\end{tabular}

Tabelle (4): Übersicht über die prozentuelle Verteilung aller Konstruktionstypen im Korpus

\section{Verfahren zur Bildung von Konversenpaaren: Medium- und Kausativderivation}

Eine große Zahl der Emotionsverben des Estnischen steht in systematischer Opposition zueinander. Sie sind semantisch äquivalent hinsichtlich ihres propositionalen Gehalts - d. h. in Bezug auf ihren Wahrheitswert gleich - und unterscheiden sich in ihrer Argumentrealisierung (Konversenbildung): Während der eine Vertreter des Oppositionspaares ein Exp/PAR-Verb ist, ist der andere ein Exp/NOM-Verb. Formal weisen beide Elemente eines Konversenpaares die gleiche verbale Wurzel auf, unterscheiden sich aber im Verbstamm hinsichtlich der Anzahl und Art der derivationellen Affixe. Ähnlich wie z. B. bei der Aktiv/Passiv-Diathese im Deutschen ist dabei auch für das Estnische zu beachten, dass sich die Konversion der Argumentrealisierung auf die Kennzeichnungsverhältnisse des Experiencers bezieht, der innerhalb eines Konversenpaares zwischen der Subjektposition und der Objektposition alterniert. Der Stimulus hingegen alterniert nicht in allen Konversenpaaren zwischen Subjekt und Objekt. Vielmehr ist eine Exp/NOM-Konverse in der Regel ein einstelliges Prädikat, bei dem der Stimulus fakultativ durch eine NP in einem Lokalkasus oder als PP angeschlossen werden kann.

Es lassen sich im Estnischen zwei Typen von Konversenpaaren etablieren. Beide werden durch Derivationsmorphologie erzeugt. Den ersten Konversentyp bilden Paare von jeweils einem Exp/NOM-Verb und einem Exp/ 
PAR\&Stim/NOM-Verb, wobei letzteres ein vom Exp/NOM-Verb abgeleitetes Kausativum ist. (17a) und (17b) illustrieren diesen Konversentyp am Beispiel des Exp/NOM-Basisverbs hirmuma 'sich fürchten' ${ }^{10}$ und des Exp/PAR\&Stim/ NOM-Kausativums hirmutama 'jmdn. ängstigen'. (17c) listet weitere dieser im Korpus nur in geringer Anzahl vertretenen Konversenpaare auf. ${ }^{11}$

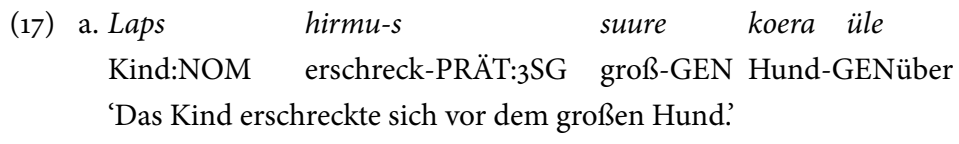

b. Lõvi hirmu-ta-s Liisa-t

Löwe:NOM erschreck-KAUS-PRÄT:3SG Liisa-PAR

oma mörigamise-ga

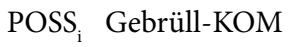

'The lion frightened Liisa with its roaring.' (Pool 1999: 26)

c. $\operatorname{Exp} / \mathrm{NOM} \quad \operatorname{Exp} / \mathrm{PAR}$

häbenema - häbendama'(be)schämen'

ehmuma - ehmatama 'erschrecken'

julguma - julgustama 'mutig werden / ermutigen'

Den zweiten Konversentyp bilden Paare, die jeweils aus einem kausativen Exp/PAR-Verb und einem davon sekundär durch REFL-Morphologie abgeleiteten Reflexiv/Medium-Verb bestehen, das dem Konstruktionstyp Exp/NOM angehört. Beispiel (18) illustriert diesen Konversentyp am Verbpaar innustama 'jmdn. begeistern', eines Exp/PAR-Verbs, s. (18a), und innustuma 'sich begeistern', eines Exp/NOM-Verbs, s. (18b). ${ }^{12}$ Weitere Verben dieses im Korpus in großer Zahl belegten Typs werden in (18c) aufgelistet ${ }^{13}$ :

(18) a. Hea hinne innus-ta-s Toomas-t veelgi rohkem gut:NOM Note:NOM begeister-KAUS-PRÄT:3SG Toomas-PAR sogar mehr 'The good grade filled Toomas with even greater enthusiasm.' (Pool 1999: 31)

b. Innus-t-u-sin selle-st idee-st begeister-KAUS-REFL-PRÄT-1SG DEM-ELA Idee-ELA 'I became enthusiastic about this idea.' (Pool 1999: 31) 
c. $\operatorname{Exp} / \mathrm{PAR} \quad \operatorname{Exp} / \mathrm{NOM}$

$\begin{array}{lll}\text { ägestama } & - \text { ägestuma } & \text { 'erhitzen, aufbrausen' } \\ \text { ärritama } & - \text { ärituma } & \text { 'verwirren' } \\ \text { erutama } & - \text { erutuma } & \text { 'aufregen' } \\ \text { hämmastama } & - \text { hämmastuma } & \text { 'erstaunen' } \\ \text { huvitama } & - \text { huvituma } & \text { 'interessieren' } \\ \text { imestama } & - \text { imestuma } & \text { 'verwundern' } \\ \text { kurvastama } & - \text { kurvastuma } & \text { 'betrüben' } \\ \text { lõbustama } & - \text { lõbustuma } & \text { 'amüsieren' } \\ \text { pahandama } & - \text { pahanduma } & \text { 'ärgern' } \\ \text { pelgama } & - \text { pelguma } & \text { 'fürchten' } \\ \text { rahustama } & - \text { rahustuma } & \text { 'beruhigen' } \\ \text { rõómustama } & - \text { rõómustuma } & \text { '(er)freuen' } \\ \text { üllatama } & - \text { üllatuma } & \text { 'überraschen' } \\ \text { vaevama } & - \text { vaevuma } & \text { 'bedrücken, plagen' } \\ \text { vihastama } & - \text { vihastuma } & \text { 'zürnen' }\end{array}$

Für einige Verben in (17c) und (18c) scheinen die Ableitungsverhältnisse aufgrund der /u/-Haltigkeit des Exp/NOM-Verbs zunächst problematisch. So könnten die Formen der Verben vaev-u-ma 'bedrücken, plagen' (18c) und pelg- $u$ - $m a$ 'scheuen, fürchten' (18c) als $u$-Ableitungen eines nominalen Stammes interpretiert werden (vaev 'Mühe', pelg 'Scheu, Furcht'), so dass sowohl das Exp/NOM- als auch das Exp/PAR-Verb als parallel aus einem Stamm abgeleitet aufgefasst werden könnte $($ vaev $\rightarrow$ vaev-u-ma). Laut Mägiste (1983: 3625) ist die detransitivierte Form jedoch von der kausativen abgeleitet, wobei der Vokal des Kausativaffixes ausfällt, also vaev $\rightarrow$ vaev-a$m a \rightarrow v a e v-u-m a$. Ein etwas problematischer Fall ist das Konversenpaar ehmuma - ehmatama 'erschrecken' in (17c). Hier ist nicht, wie in den anderen Fällen dieses Konversentyps, das Exp/NOM-Verb direkt die Basis der Kausativderivation, da das kausative Exp/PAR auf den Stamm ehma- auslautet und daher nicht auf den Stamm des Exp/NOM-Verbs - ehmu- - zurückzuführen ist. Beide Verben des Konversenpaares teilen sich mithin die Wurzel, nicht jedoch den Stamm. Bei allen anderen Konversenpaaren meines Korpus entspricht der Stamm des derivierten Verbs ohne Derivationsaffix dem Stamm der Derivationsbasis. 
Im Ergebnis ist festzuhalten, dass eine Vielzahl estnischer Emotionsverben hinsichtlich des Konstruktionstyps in systematischer morphologischer Opposition zueinander steht (morphosyntaktische Konverse). Diese Konversenpaare werden durch die Verwendung der Teilsysteme zur Valenzerweiterung (KAUS) und zur Valenzreduktion (REFL) des im Estnischen vorhandenen ausdifferenzierten Systems der Derivationsmorphologie erzeugt. Es lassen sich daher die zwei Typen von Konversenpaaren etablieren:

Exp/NOM-Basisverb $\leftrightarrow$ Exp/PAR-Verb mit KAUS

Exp/PAR-Verb mit KAUS $\leftrightarrow$ Exp/NOM-Verb mit KAUS und REFL

Aufgrund dieser morphologischen Teilsysteme zur Bildung von Konversenpaaren ist das Estnische mithin gut geeignet, die in der Forschung zur Argumentrealisierung von Emotionsverben prominente Kausalstrukturthese zu überprüfen.

\section{Zur Kausalstrukturthese}

Die für die Diskussion der Konstruktionsvarianz bei Emotionsverben einschlägigen Theorien basieren auf der Annahme, dass die an kanonischen Handlungsverben (z. B. dt. zerstören, töten) entwickelten Mechanismen auf die Argumentrealisierung von Emotionsverben übertragbar seien. Dies gilt sowohl für die semantischen als auch für die kognitiv ausgerichteten Modelle. So wird bspw. in mit semantischen Rollenhierarchien arbeitenden Theorien die Rollensemantik der Emotionsverben in die Rollensemantik der Handlungsverben eingepasst, indem Stimulus-Partizipanten in Subjektposition als Causer aufgefasst werden (z. B. Pesetzky 1995: 55). In mit CAUSE-Operator arbeitenden dekompositionellen Modellen werden Emotionsverben in solche mit kausativer und solche mit nicht-kausativer semantischer Struktur klassifiziert (z. B. Iwata 1995; Van Valin/LaPolla 1997: 102-129). In kognitiven Ansätzen wird die Kausalrelation, die zwischen Partizipanten von Sachverhaltsdenotationen der physischen Domäne gilt, als prototypische Vorlage erachtet, in das Emotionsverben koerziert werden (Langacker 1991: 221f.; Croft 1991: 212-215).

Aus der Annahme eines kausalen Unterschieds zwischen verschiedenen Konstruktionstypen von Emotionsverben ergibt sich zum einen die Schwierigkeit, dass (mit Ausnahme der Role and Reference Grammar und Croft 1991, 1993) lediglich zwei Klassen von Emotionsverben berücksich- 
tigt werden: sogenannte Subjekt- und Objekt-Experiencer-Verben. In einer Reihe einschlägiger Theorien (u. a. Grimshaw 1990; Dowty 1991; Iwata 1995; DeDisidero 1999) wird ein in vielen Sprachen verbreiteter Konstruktionstyp mit Exp/DAT und der für das Estnische relevante Typ mit Exp/LOK ausgeblendet bzw. als Objekt-Experiencer-Konstruktionstyp behandelt und somit nicht vom Konstruktionstyp Exp/AKK unterschieden. Beide Typen - Exp/DAT bzw. Exp/LOK und Exp/AKK - realisieren den Stimulus in Subjektposition; demnach sollte für beide die gleiche theoretische Annahme in Bezug auf die Realisierungsmechanismen für den Stimulus (Anhebung seiner Position in der dekomponierten Prädikatsstruktur durch den CAUSE-Operator bzw. Anhebung seiner Position in der Rollenhierachie von Thema zu Causer) gelten.

Die Kausalstrukturthese (u. a. Grimshaw 1990; Iwata 1995; DiDesidero 1999; Pesetzky 1995) geht davon aus, dass die Argumentrealisierung von Verben mit Experiencer in Objektposition auf eine kausative Bedeutungskomponente dieser Verben zurückzuführen ist, die diesen Konstruktionstyp vom Subjekt-Experiencer-Typ, der als nicht-kausativ aufgefasst wird, unterscheidet. Da die ostseefinnischen Sprachen über morphologische Mittel der Derivation kausativer Emotionsverben verfügen, eignen sie sich gut zur Überprüfung des Kausalstrukturmodells. Für das Finnische liegen bisher zwei Arbeiten zur Kausalität und Argumentrealisierung von Emotionsverben vor (Pylkkänen 2000; Nelson 2003). Diese setzen sich vor allem mit der Kausalstrukturthese Pesetskys (1995) auseinander. Die Argumentation beider Arbeiten konzentriert sich dabei vor allem auf einen Wortbildungstyp des Finnischen, nämlich die Derivation kausativer Verben von inchoativen Verben (z. B. viha-stu- angry-INCH 'become angry' > viha-stu-tta- angry-INCH-CAUS 'cause to become angry', Pylkkänen 2000: 419), der im Estnischen keine Entsprechung hat. Trotz der nahen Verwandtschaft unterscheiden sich beide Sprachen hinsichtlich des Inventars verbaler Derivationsmorphologie erheblich (vgl. Kasik 1997). Befunde zur Kausalstrukturthese, wie sie das Finnische zeigt, sind mithin nicht unbedingt im Estnischen zu erwarten.

Fast alle Exp/PAR-Verben, die in meinem Korpus enthalten sind, tragen Kausativmorphologie, mit einer einzigen Ausnahme, dem Simplexverb härima 'stören'. Damit scheinen die Derivationsverhältnisse des Estnischen die Kausalstrukturthese zunächst zu bestätigen.

Bei einer genaueren Untersuchung größerer Datenmengen des Estnischen zeigt sich allerdings, dass es eine kleine Zahl von Exp/NOM-Verben 
gibt, die ebenfalls Kausativmorphologie aufweisen. Das Verb armastama 'lieben, mögen', das in (19) angeführt ist, illustriert diesen Wortbildungstyp.

(19) armastama 'lieben, mögen' $\leftarrow$ armas 'lieb' + -ta 'KAUS'

Aarne ei armas-ta füüsili-st tö̈-d
Aarne NEG lieb-KAUS physisch-PAR Arbeit-PAR
Aarne mag keine körperliche Arbeit.' (MU: 1,48)

Zwar ist die Derivation der Verben von nominalen Stämmen nicht immer transparent. Aber die in der vorliegenden Arbeit untersuchten Verben werden in der Forschungsliteratur eindeutig als Kausativderivationen zu nominalen Stämmen identifiziert (s. Viitso 2003: 72f; Vainik 2001: 45). Das Exp/ NOM\&Stim/PAR-Verb armastama wird als Faktitiv-Ableitung vom Adjektiv armas 'lieb' aufgefasst. Gleiches gilt für die Exp/NOM\& Stim/PAR-Verben vihkama 'hassen' als denominale Derivation von viha 'Wut' (vgl. auch Mägiste 1983: 3809). Ein weiterer Kandidat für diesen Wortbildungstyp sind die Exp/NOM-Verben võorastama 'fremdeln' mit der adjektivischen Basis võõras 'fremd' und austama 'respektieren' mit der adjektivischen Basis aus 'ehrenhaft'. Das ebenfalls eindeutig als kausatives Verb zu identifizierende rõõmustama '(er)freuen' (Viitso 2003: 72; EKG I: 429)) kann einerseits als transitives Stim/NOM\&Exp/PAR-Verb (20a) verwendet werden und ist Teil eines Konversenpaares rõõmustama - rõõmustuma (vgl. 18c).

(20) a. Mees

$$
\text { rõõmus-ta-b nai-st lille-de-ga }
$$

Mann:NOM freu-KAUS-PRS:3SG Frau-PAR Blume-PL-KOM

'Der Mann erfreut die Frau mit Blumen.' (EKSS: 206)

b. Päike rõomus-ta-b heinalisi

Sonne freu-KAUS-PRS:3SG Heumäher-PAR:PL

'Die Sonne erfreut die Heumäher.' (EKSS: 206)

Zum anderen ist es jedoch auch als Exp/NOM-Verb verwendbar. In dieser Verwendung steht es mit Komplementsatz (21a, 21b).
(21) a. Rõõmus-ta-b,
et talv
on
läbi
freu-KAUS-PRS:3SG KOMP Winter:NOM sei:PRS:3SG durch
'Er/Sie freut sich, dass der Winter vorbei ist.' (EKS: 206) 
b. Ja suur inimene rõomus-ta-s väga, (...)

und groß:NOM Mensch:NOM freu-KAUS-PRÄT:3SG sehr

'Und der große Mensch freute sich sehr, (dass er einen so vernünftigen Mann kennengelernt hatte.)' (VP 1,31)

Das formal kausative Verb rõõmustama '(er)freuen' kann also in zwei unterschiedlichen Konstruktionen stehen, die jeweils eine unterschiedliche Argumentrealisierung des Experiencers bedingen. Vergleichbares gilt für das Verb imestama 'wundern', einer kausativen Ableitung mit der nominalen Basis ime 'Wunder'14. Es kann einerseits als transitives Stim/NOM\&Exp/ PAR-Verb (22a) verwendet werden und ist Teil eines Konversenpaares imestama - imestuma (vgl. 22b).

(22) a. See

$$
\text { ei imesta meid sugugi }
$$

DEM:NOM NEG wunder 1 PL:PAR überhaupt

'Das verwundert uns nicht im Mindesten.' (Saagpakk 1992: 173)

b. $\mathrm{Ma} \quad$ imestun kuul-de-s, et (...)

1SG:NOM wunder-1SG hör-INF-INE KOMP

'Es wundert mich zu hören, dass ...' (Saagpakk 1992: 173)

Zum anderen ist es jedoch auch als Exp/NOM-Verb verwendbar. In dieser Verwendung regiert es die Postposition üle 'über', vgl. (23).

(23) $\mathrm{Ma}$ ei imesta enam millegi üle 1SG:NOM NEG wunder mehr was:GEN über 'Ich wundere mich über nichts mehr'. (Pool 1990: 30)

Hinsichtlich der Kausalstrukturhypothese ist mithin zusammenfassend festzuhalten, dass im Estnischen zwar eine große Mehrheit der kausativen Emotionsverben ihr Stimulus-Argument syntaktisch als Subjekt realisiert. Gleichzeitig gibt es jedoch eine kleine Gruppe kausativer Emotionsverben, die ihr Experiencer-Argument als Subjekt realisiert. Für das Estnische kann daher, anders als von der Kausalstrukturhypothese erwartet, kein direkter Zusammenhang von kausativer Morphologie des Emotionsverbs und der Realisierung des Stimulus in der Subjektposition festgestellt werden. 


\section{Ergebnis}

In der einschlägigen sich mehrheitlich auf die Verhältnisse des Englischen konzentrierenden Forschung zu Emotionsverben werden diese hinsichtlich ihres Argumentrealisierungverhaltens gemeinhin in zwei Klassen, nämlich solche, die den Experiencer und solche die den Stimulus als Subjekt realisieren, aufgespalten. Wie die hier vorgelegte Untersuchung zum Estnischen gezeigt hat, ist übereinzelsprachlich von einer sehr viel größeren als der in der einschlägigen Forschung erfassten Konstruktionsvielfalt auszugehen (vgl. dazu auch Kutscher 2009 für die Konstruktionsvielfalt bei Emotionsverben des Deutschen, Türkischen, Baskischen und Lasischen, sowie Kailuweit 2005 für Italienisch und Französisch). Eine Mehrzahl der von mir analysierten Verben verteilt sich zwar auf den in der Forschungsliteratur meistdiskutierten Kasusrahmen Exp/NOM\&Stimulus/PAR (Subjekt-Experiencer-Verben) bzw. umgekehrt Stimulus/NOM\&Exp/PAR (Objekt-Experiencer-Verben). Darüber hinaus verfügt das Estnische aber über eine Reihe weiterer Konstruktionstypen, wie ausführlich in Abschnitt 3 dargestellt wurde.

Hinsichtlich der in der Literatur statuierten Korrelation zwischen Kausativmorphologie und Konstruktionstyp <Stimulus in Subjektposition $>$ (u. a. Grimshaw 1991: 23; Pesetzky 1995: 63-69; Pylkkänen 2000: 418; Van Valin/LaPolla 1997: 98) wurde in Abschnitt 4 gezeigt, dass sich diese Annahme für das Estnische in der aus theoretischen Gründen erforderlichen Ausschließlichkeit nicht bestätigt. Zwar tragen mit Ausnahme eines einzigen Verbs alle Exp/ PAR-Verben des Estnischen Kausativmorphologie. Bei einer genaueren Untersuchung größerer Datenmengen dieser Sprache zeigt sich allerdings, dass es eine kleinere Zahl von Exp/NOM-Verben gibt, die ebenfalls Kausativmorphologie aufweisen. Für das Estnische kann daher kein direkter Zusammenhang von kausativer Morphologie des Emotionsverbs und der Realisierung des Stimulus als Subjekt festgestellt werden. Der Befund zur Konstruktionsvielfalt estnischer Emotionsverben liefert somit Ergebnisse, die für eine Verfeinerung der linguisitschen Theoriebildung berücksichtigt werden müssen.

Silvia Kutscher Institut für Allgemeine Sprachwissenschaft Westfälische Wilhelms-Universität Münster silvia.kutscher@culture.hu-berlin.de 


\section{Silvia Kutscher}

\section{Anmerkungen}

1 Ein anonymer Gutachter merkt an, dass sich in den aufgeführten Sprachbeispielen auch einige Verben finden, die als im Gebrauch selten im EKSS vermerkt sind. Da die vorliegende Untersuchung auf eine Analyse der Argumentrealisierungstypen abzielt, ist dies nicht als problematisch zu bewerten. Die morphosyntaktischen Eigenschaften der entsprechenden Verben sind unbestritten und im Rahmen des grammatischen Systems des Estnischen nicht auffällig.

2 Die Distribution der unter (3b) und (3c) illustrierten Suffixe in ihrer interkategoriellen derivativen Funktion ist laut Kasik (1997: 54) phonologisch determiniert. Ihrer Ansicht nach tritt das hier als REFL glossierte Derivationsmorphem an monosyllabische nominale Stämme (3b), während das hier als KAUS glossierte Morphem an polysyllabische nominale Stämme affigiert wird (3c). Mägiste (1983, passim) sieht allerdings einige Verben als Kausativderivation von monosyllabischen nominalen Basen.

3 So kann das Derivationsmorphem $-u$ sowohl denominal derivieren (all- $u-m a$, (3a)) als auch intrakategoriell valenzreduzierend wirken (rikast-u-ma, (4b)). Basierend auf der theoretischen Annahme, dass Homonymie in der morphologischen Analyse möglichst vermieden werden sollte, wird im vorliegenden Artikel davon ausgegangen, dass in (3a) und (4b) nicht zwei homonyme Derivationsmorpheme vorliegen, sondern dass es sich um ein Morphem handelt: Sowohl bei der denominalen als auch bei der deverbalen Ableitung mit dem Morphem $-u$ ist das Ergebnis ein reflexiv-passives Verb. Diese Gemeinsamkeit soll sich in der von mir verwendeten Glossierung widerspiegeln: Das Morphem $-u$ wird stets durch eine einheitliche Glosse REFL gekennzeichnet. Diesem Vorgehen liegt mithin die theoretische Annahme zugrunde, dass die einheitlich glossierten Morpheme jeweils polysem sind und in Abhängigkeit von der jeweiligen Ableitungsbasis unterschiedliche Funktionen ausüben. Für das Derivationssuffix - $t a$ wurde aus vergleichbaren Gründen KAUS glossiert. Die unterschiedlichen Funktionen dieses Morphems lassen sich als Valenzerweiterung generalisieren, vgl. auch Fußnote 6.

4 Laut Viitso (2003: 73) liegt mit valetama eine instruktive Derivation vor. Nach dieser Auffassung wäre das Verb semantisch zu interpretieren als Bezeichnung für einen Vorgang, bei dem jemand mit einer Lüge versehen wird. M.E. ist die faktitive Analyse des Verbs valetama vorzuziehen, da es ja die Handlung des Lügens selbst bezeichnet, also den Prozess der Erzeugung einer Lüge.

5 Der Partitiv Plural lautet kirju.

6 Die logisch-semantische Funktion des Kausativkennzeichens ist in beiden Fällen gleich: bei der Kausation eines Verbs wird die Valenz erhöht, während sie bei der denominalen Ableitung Valenz erzeugt. In beiden Fällen werden der Ableitungsbasis Argumentstellen hinzugefügt (s. auch Kulikov 2001:886).

7 Neben den hier dargestellten Funktionen kann eine -ta-Ableitung auch noch instrumentale und essentiale Verben erzeugen. Im ersteren Fall drückt das abgeleitete Verb aus, dass das vom nominalen Stamm bezeichnete Objekt als Instrument einer Handlung dient (z. B. ahel-da-ma 'anketten' $\leftarrow$ ahel 'Kette'), im letzteren Fall drückt es aus, dass eine Handlung vollzogen wird, die typisch für die von der nominalen 
Basis bezeichnete Entität ist (z. B. varas-ta-ma 'stehlen' $\leftarrow$ varas 'Dieb'), vgl. Viitso 2003: 72ff.). Beide Ableitungstypen sind für die zu analysierenden Daten der vorliegenden Arbeit nicht von Relevanz, werden aber von mir ebenfalls als semantische Subtypen der morphologisch definierten Gruppe der Kausativa aufgefasst.

8 Reflexivkonstruktionen im engeren Sinne - also Sachverhalte, die eine Handlung denotieren, welche ein kontrollierendes Agens an sich selbst ausführt - werden allerdings häufig periphrastisch durch Verwendung des Reflexivpronomens ise gebildet, s. z. B. ennast pesema 'sich waschen' in (Ib).
(I) a. Isa
pese- $b$
auto-t
Vater:NOM wasch:PRS-3SG Auto-PAR
'Der Vater wäscht das Auto.' (EKSS: 276)
b. Isa pese-b ennast sauna-s
Vater:NOM wasch:PRS-3SG ise:PAR Sauna-INE
'Der Vater wäscht sich in der Sauna.' (EKSS:275)

Nur für einen Teil der Verben ist auch die Bildung der Reflexivvariante durch Ableitung mit $-u$ möglich (vgl. riietuma in (5b)), andere erlauben nur die Bildung des periphrastischen Reflexivs. So ist es z. B. nicht möglich, eine Reflexivform von pese$m a$ 'waschen' (s. (II)) durch - $u$-Ableitung zu bilden.

(II) *pes-u-ma 'wasch-REFL-INF'

9 Ein anonymer Gutachter verweist auf kontrastive Studien zu Emotionsprädikaten im Finnischen und Estnischen (Tiia Palosaar) bzw. im Deutschen und Estnischen (Ani Rääts), die gerade erstellt werden. Diese sind mir jedoch nicht zugänglich gewesen.

10 Für das Exp/NOM-Verb hirmuma 'fürchten' ist davon auszugehen, dass es sich um eine denominale Nullableitung vom Vokalstamm des Nomens hirm 'Furcht:NOM' handelt, der auf /u/ auslautet: hirmu 'Furcht:GEN'. Diese Annahme wird unterstützt durch Viitso (2003: 73). Dort wird die Kausativableitung hirmu-ta-ma 'jmdn. ängstigen' mit Verweis auf den Vokalstamm des Nomens (hirmu) als denominale Kausativierung aufgefasst.

11 Von den in (17c) aufgeführten Verben werden zwei von einem anonymen Gutachter als eher okkasionelle, literarische Bildungen beurteilt (julguma, häbendema). Da beide Verben in Wörterbüchern des Estnischen aufgeführt werden (julguma: Saagpakk, Kann et al. häbendama: Saagpakk), wurden sie in der vorliegenden Arbeit als im estnischen Wortschatz etablierte Verben erachtet und daher nicht aus dem Korpus genommen.

12 Das Exp/NOM-Verb innustuma ist eine Detransitivierung des kausativen Exp/PARVerbs innustama. Das /a/ des Kausativaffixes wird durch die Detransitivierung getilgt, vgl. auch (3b).

13 Von den in (18c) aufgeführten Verben werden drei von einem anonymen Gutachter als eher okkasionelle bzw. literarische Bildungen beurteilt (ägestama, hämmastuma, imestuma). Da alle Verben jeweils in den Wörterbüchern Saagpakk und Kann et al. aufgeführt werden, wurden sie in der vorliegenden Arbeit als im estnischen Wortschatz etablierte Verben erachtet und daher nicht aus dem Korpus genommen.

14 Das Nomen hat im Partitiv Plural (noch) einen s-haltigen Stamm: imes-id 'WunderPAR:PL'. 


\section{Abkürzungen}

$\begin{array}{llll}\text { ABL } & \text { Ablativ } & \text { NEG } & \text { Negation } \\ \text { ADE } & \text { Adessiv } & \text { NOM } & \text { Nominativ } \\ \text { Ag } & \text { Agens } & \text { NOM'er } & \text { Nominalisierer } \\ \text { ALL } & \text { Allativ } & \text { OBJ } & \text { Objekt } \\ \text { AKK } & \text { Akkusativ } & \text { PAR } & \text { Partitiv } \\ \text { AUX } & \text { Auxiliar } & \text { Pat } & \text { Patiens } \\ \text { BEN } & \text { Benefaktiv/Benefiziens } & \text { PL } & \text { Plural } \\ \text { DAT } & \text { Dativ } & \text { POSS } & \text { Possessiv } \\ \text { DEM } & \text { Demonstrativum } & \text { PP } & \text { Adpositionalphrase } \\ \text { ELA } & \text { Elativ } & \text { PRÄT } & \text { Präteritum } \\ \text { ESS } & \text { Essiv } & \text { PRS } & \text { Präsens } \\ \text { Exp } & \text { Experiencer } & \text { PRV } & \text { Präverb } \\ \text { GEN } & \text { Genitiv } & \text { PTZP } & \text { Partizip } \\ \text { ILL } & \text { Illativ } & \text { QU } & \text { Interrogativ } \\ \text { IMP } & \text { Imperativ } & \text { REC } & \text { Rezipient } \\ \text { INE } & \text { Inessiv } & \text { REFL } & \text { Reflexiv-Passiv, } \\ \text { INF } & \text { Infinitiv } & & \text { Detransitivierung } \\ \text { INS } & \text { Instrumental } & \text { REL } & \text { Relativpronomen } \\ \text { KAUS } & \text { Kausativ } & \text { SBJ } & \text { Subjekt } \\ \text { KOM } & \text { Komitativ } & \text { SG } & \text { Singular } \\ \text { KOMP } & \text { Kennzeichnung für } & \text { Stim } & \text { Stimulus } \\ & \text { Komplementsätze } & \text { SUB } & \text { Subordinierer } \\ \text { KONNEG } & \text { Konnegativ } & \text { TRA } & \text { Translativ } \\ \text { LOK } & \text { Kasus der inneren und } & & \\ & \text { äußeren Lokalkasusreihen } & & \\ & & & \end{array}$

\section{Datenquellen}

EKG = Erelt, Mati \& Kasik, Reet \& Metslang, Helle \& Rajandi, H. \& Ross, K. \&

SAARI, H. \& TAeL, K. \& VARE, S. 1995: Eesti keele grammatika I: Morfoloogia, sõnamoodustus. Tallinn.

EKSS = RAIET, E. et al. (eds.) 1988-2002: Eesti Kirjakeele Seletussõnaraamat. 6 Bde. Tallinn.

Kann, K. \& Kibbermann, E. \& Kibbermann, F. \& Kirotar, S. 1972: Eesti-saksa sõnaraamat. 3. Aufl. Tallinn.

Kibbermann, E. \& Kirotar, S. \& Koppel, P. 1975: Saksa-eesti sõnaraamat. Tallinn.

MäGISTE, Julius 1983: Estnisches etymologisches Wörterbuch. 12 Bände. Helsinki.

PoOL, RaIli 1999: Eesti keele verbirektsioone. Tartu.

SaAgPakK, Paul F. 1992: Eesti-Inglise sõnaraamat. 2. Aufl. Tallinn.

VP = SAINT-ExUPÉRY, ANTOINE DE 1993: Väike prints. Übersetzt von Ott Ojamaa. Tallinn.

MU = UnT, Mati 1992: Tere, kollane kass! Tallinn. 


\section{Literaturverzeichnis}

Belletti, Adriana \& Rizzi, Luigi 1988: Psych-Verbs and Theta-Theory. - Natural Language and Linguistic Theory 6. 291-352.

Croft, William 1991: Syntactic Categories and Grammatical Relations. The Cognitive Organization of Information. Chicago.

- 1993: Case marking and the semantics of mental verbs. - Semantics and the Lexicon. 55-72. Hrsg. von J. Pustejovsky. Dordrecht.

DiDesidero, Linda B. 1999: Psych Verbs. Acquisition, Lexical Semantics, and Event Structure. Dissertation, Northwestern University at Evanston, Illinois. Evanston, Illinois.

Dowty, David R. 1991: Thematic proto-roles and argument selection. - Language 67. 547-619.

Grimshaw, Jane B. 1990: Argument Structure. Cambridge, MA.

HAKUlinen, LAURI 1957: Handbuch der finnischen Sprache. Wiesbaden.

HärTL, HOLDEN 1999: fürchten vs. ängstigen: Thematische Rollen und Ereignisstrukturen psychischer Verben in einem Modell der Sprachproduktion. - Proceedings der 4. Fachtagung der Gesellschaft für Kognitionswissenschaft, Bielefeld, 28. September 1. Oktober 1999. 189-194. Hrsg. von I. Wachsmuth, B. Jung. Sankt Augustin.

- 2001: CAUSE and CHANGE. Thematische Relationen und Ereignisstrukturen in Konzeptualisierung und Grammatikalisierung. Studia grammatica 50. Berlin.

IwATA, SEIzI 1995: The distinctive character of psych-verbs as causatives. - Linguistic Analysis 25. 95-120.

KAILUWEIT, RoLF 2005: Linking Syntax und Semantik französischer und italienischer Gefühlsverben. Linguistische Arbeiten 493. Tübingen.

KASIK, REET 1997: Typology of Estonian and Finnish word formation. - Estonian language. Hrsg. von Mati Erelt. Linguistica Uralica. Supplementary Series 1. Tallinn. $42-72$.

Kazenin, Konstantin I. 2001. Verbal reflexives and the middle voice. - Language typology and Language Universals. 916-927. Hrsg. von Martin Haspelmath, Ekkehard König, Wulf Oesterreicher, Wolfgang Raible. Handbücher zur Sprach- und Kommunikationswissenschaft 20.1. Berlin.

Kemmer, Suzanne 1993. The middle voice. Typological Studies in Language 23. Amsterdam.

Klein, Nina \& Kutscher, Silvia 2002: Psych-verbs in German and the Principle of Lexical Economy. Arbeitspapiere des SFB 282 Theorie des Lexikons 122. Düsseldorf.

- 2005: Lexical economy and case selection of psych-verbs in German. Überarb. Version von Klein/Kutscher 2002.

http://www.linguistics.ruhrunibochum.de/ klein/papers/lexEconPsych.pdf.

Kulikov, Leonid I. 2001: Causatives. - Language Typology and Language Universals. 886-898. Hrsg. von Martin Haspelmath, Ekkehard König, Wulf Oesterreicher, Wolfgang Raible. Handbücher zur Sprach- und Kommunikationswissenschaft 20.1. Berlin.

Kutscher, Silvia 2009: Kausalität und Argumentrealisierung. Zur Konstruktionsvarianz bei Psychverben in europäischen Sprachen. Linguistische Arbeiten 528. Tübingen. 


\section{Silvia Kutscher}

LaAnest, ARvo 1982: Einführung in die ostseefinnischen Sprachen. Gegenüber der estn. Ausg. von 1975 vom Verf. verb. u. erg. autorisierte Übertragung aus dem Estn. von Hans-Hermann Bartens. Hamburg.

Langacker, Ronald W. 1987: Foundations of Cognitive Grammar. Vol. 1: Theoretical Prerequisites. Foundations of Cognitive Grammar 1. Standford.

- 1991: Concept, Image, and Symbol. The Cognitive Basis of Grammar. Cognitive linguistics research 1. Berlin.

LYONs, JoHN 1968. Introduction to theoretical linguistics. Cambridge.

Nelson, Diane 2003: Case and event structures in Finnish psych predicates. - New perspectives in Case Theory. 191-221. Hrsg. von Ellen Brandner, Heike Zinsmeister. Stanford.

Pesetsky, David 1995: Zero Syntax: Experiencers and Cascades. Current Studies in Linguistics 27. Cambridge, Mass.

Postal, Paul M. 1971: Cross Over Phenomena. New York.

PyLKKäNEn, LIINA 2000: On stativity and causation. - Events as grammatical objects. The converging perspectives of lexical semantics and syntax. Hrsg. von C. Tenny, J. Pustejovsky. 417-442. CSLI Lecture Notes. Stanford.

VAINIK, EnE 2001: Eestlaste Emotsioonisõnavara. Magisterarbeit. Tallinn: Eesti Keele Instituut.

- 2002: 'Hot-blooded' Estonians. On Estonian's folk category of emotions. http://www.folklore.ee/folklore/vol21/emotions.pdf [letzter Zugriff: 11.05.2011].

VAN VAlin, Robert. D. \& La Polla, Randy J. 1997: Syntax: structure, meaning and function. Cambridge textbooks in linguistics. Cambridge.

Virtso, Tirt-Rein 2003: Structure of the Estonian language. Phonology, morphology and word-formation. - Estonian language. 9-92. Hrsg. von Mati Erelt. Linguistica Uralica. Supplementary Series 1. Tallinn. 